\title{
Differential Spontaneous Photon Emissions from Cerebral Hemispheres of Fixed Human Brains: Asymmetric Coupling to Geomagnetic Activity and Potentials for Examining Post-Mortem Intrinsic Photon Information
}

\author{
Justin N. Costa ${ }^{1,2}$, Nicolas Rouleau ${ }^{2,3}$, Michael A. Persinger ${ }^{1,2,3 *}$ \\ ${ }^{1}$ Department of Biology, Laurentian University, Sudbury, Canada \\ ${ }^{2}$ Behavioural Neuroscience, Laurentian University, Sudbury, Canada \\ ${ }^{3}$ Biomolecular Sciences Programs, Laurentian University, Sudbury, Canada \\ Email: mpersinger@laurentian.ca
}

Received 13 April 2016; accepted 24 May 2016; published 27 May 2016

Copyright (C) 2016 by authors and Scientific Research Publishing Inc.

This work is licensed under the Creative Commons Attribution International License (CC BY). http://creativecommons.org/licenses/by/4.0/

\section{(c) (i) Open Access}

\section{Abstract}

The emissions of biophotons have been considered a ubiquitous property of living systems and their components. We measured the "spontaneous" photon emissions from fixed whole and sectioned human brains within hyper-dark settings. Significant differences in photon counts were measured from different spatial planes. The flux densities were in the order of $2 \times 10^{-12} \mathrm{~W}^{\text {per }} \mathrm{m}^{2}$. The right hemispheres but not the left hemispheres displayed more photon emissions whose spectral power density profiles exhibited a conspicuous amplitude peak between 7.9 and $8 \mathrm{~Hz}$. Brains measured in the hyperdark $\left(\sim 10^{-12} \mathrm{~W} \cdot \mathrm{m}^{-2}\right)$ after removal from the typical lighting of the laboratory emitted more photons than those that had been maintained in the hyperdark for one week. The significant correlation between the numbers of photons emitted from the left hemisphere (but not the right) and global geomagnetic activity also exhibited energy equivalence between the photon flux densities and the geomagnetic shift within the cerebral volumes. These results indicate that what has been assumed to be fixed unresponsive human brain tissue still emits small numbers of photons that may be residuals from ambient light and can potentially interact with global geomagnetic activity. The medical implications for post-mortem intrinsic photonic information based upon the anisotropic microstructures within the hemispheres of the human cerebrum are discussed.

${ }^{*}$ Corresponding author.

How to cite this paper: Costa, J.N., Rouleau, N. and Persinger, M.A. (2016) Differential Spontaneous Photon Emissions from Cerebral Hemispheres of Fixed Human Brains: Asymmetric Coupling to Geomagnetic Activity and Potentials for Examining Post-Mortem Intrinsic Photon Information. Neuroscience \& Medicine, 7, 49-59. http://dx.doi.org/10.4236/nm.2016.72006 
Keywords

Photons, Fixed Human Brain, Geomagnetic Activity, Hemispheric Differences, Ambient Light

\section{Introduction}

The emissions of photons from living systems have been examined and verified quantitatively from multiple perspectives. The perspicacious formulations by Popp and his colleagues [1]-[3] concerning the physical and chemical mechanisms for biophoton emissions have been demonstrated in other contexts. Photon counts near living tissue that exceed background flux densities have been measured from bacteria [4], cells [5], hippocampal slices [6], and the brains of human subjects engaging in specific cognitions [7] [8]. This list is by no means exhaustive. The order of magnitude of these photon emissions is $\sim 10^{-12} \mathrm{~W} \cdot \mathrm{m}^{-2}$. The ubiquitous nature of photon emissions from likely multiple mechanisms [9] within the living system suggests they may be more directly involved with communication between living units such as cells and bacteria [10] [11]. Communication also implies the containment and generation of information.

However, to our knowledge, there have been no studies that have examined the spontaneous photon emissions from fixed human brains. When fixed properly, the micromorphological detail of the cerebral cortices remains remarkably intact for decades as verified by histological examination at $1000 \times$. For several years we have measured a "background" radiant flux density by a variety of digital and analogue photomultiplier units that discern an order of magnitude of $\sim 10^{-12}$ to $10^{-11} \mathrm{~W} \cdot \mathrm{m}^{-2}$ [12] [13]. These values are about 10 to 100 times greater than the estimated values for cosmic ray densities at ground level [14]. In this article, we showed that spontaneous photon emissions increased near fixed whole brains and sections and that the power spectra of the amplitude modulations of these flux densities were not random and may reflect the capacity to retain photonic energies.

\section{Materials \& Methods}

\subsection{Photomultiplier Tube}

A single photomultiplier tube (PMT) from Sens-Tech Sensor Technologies (Model DM0090C) was used in all experiments to measure temporal photon counts. The spectral response range was between $300-850 \mathrm{~nm}$. Sens-Tech Counter Timer software recorded all data with a $50 \mathrm{~Hz}$ sampling rate for 5000 readings ( $20 \mathrm{msec}$ data points for 100 seconds) on a Lenovo ThinkPad laptop. All measurements were taken within a cardboard box (7.5 $\mathrm{cm} \times 72 \mathrm{~cm} \times 51 \mathrm{~cm}$ ) in which the brain samples and photomultiplier sensor unit were placed. All trials occurred within a hyperdark enclosed chamber, with USB connections from the photomultiplier unit connected through to an external room where the data-logging laptop was positioned.

\subsection{Specimens}

Photon counts were obtained from preserved whole-brains $(\mathrm{n}=3)$, sagittal brain portions of both left $(\mathrm{n}=2)$ and right $(n=2)$ hemispheres, and coronal brain slices $(n=3$; different brain sources) that had been stored in the Neurochemistry laboratories for the Behavioural Neuroscience Program at Laurentian University. Whole brains were either light-deprived for 7 days or remained within a laboratory that was illuminated by overhead fluorescent lights (250 lux) during storage and between trials. The flux in front and behind the containers ranged from 5 lux to 50 lux. All specimens were removed from their plastic storage containers containing EFA (ethanol-formalin-acetic acid) and placed into the box on folded paper towels. Whole and sagittal preparations were placed into the box directly. Coronal slices were placed in a tissue culture dish $(15 \mathrm{~cm}$ in diameter) filled with EFA solution and were wrapped in a black nitrile glove to cover one hemisphere for measurements of each exposed hemisphere individually.

\subsection{Procedure}

Whole brains were placed into the cardboard storage box (Figure 1) approximately $9 \mathrm{~cm}$ from the aperture of the photomultiplier unit. Six aspects of the whole brains were used to record the photon counts: rostral, caudal, 
left, right, dorsal, and ventral aspects (Figure 2). When recording the dorsal and ventral aspects of the whole brain, the photomultiplier unit was suspended and placed onto supporting structures along the top of the box. The aperture of the PMT faced down onto the brain at a $9 \mathrm{~cm}$ distance. The whole brain was placed on its dorsal surface facing down (ventral aspect upwards) when recording the ventral aspect of the whole brain. Baseline recordings were taken with no brain present in the box and with the photomultiplier aperture facing downward into the box.

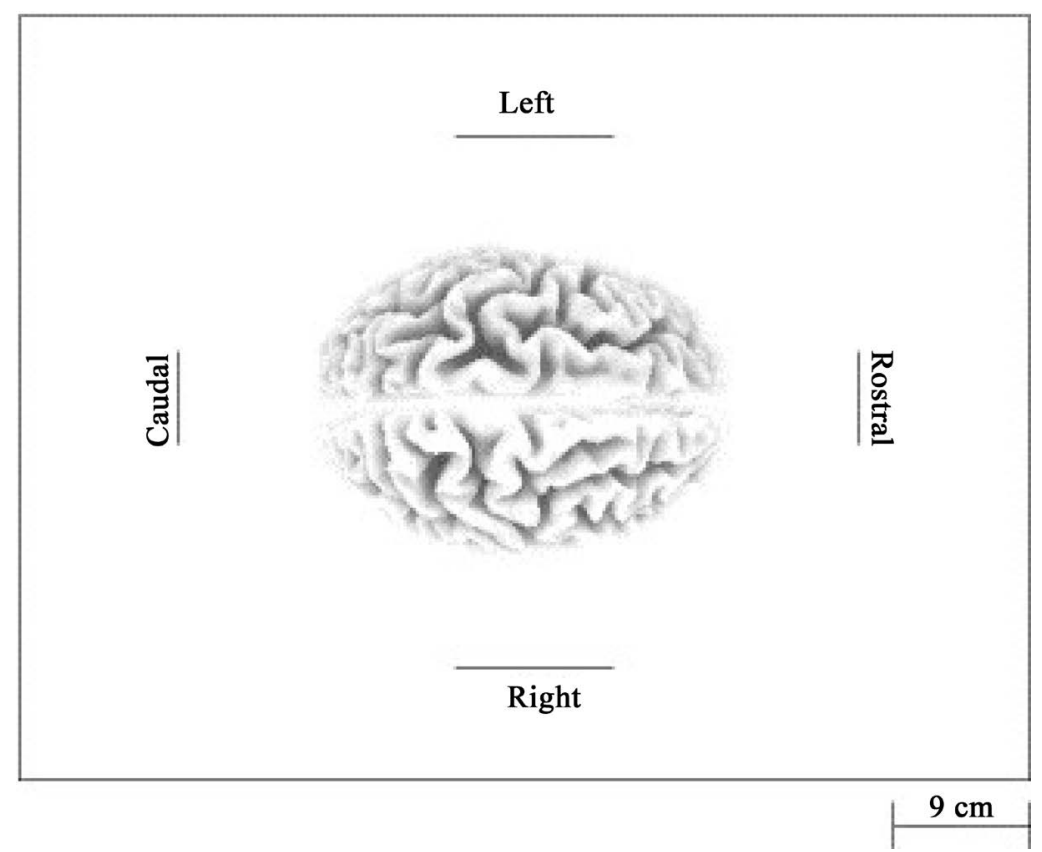

Figure 1. Dorsal view of the whole-brain placed within a box. Spatial orientation is provided where lines indicate the position and orientation of the PMT aperture. A $9 \mathrm{~cm}$ buffer zone was maintained between the brain and the measurement device.

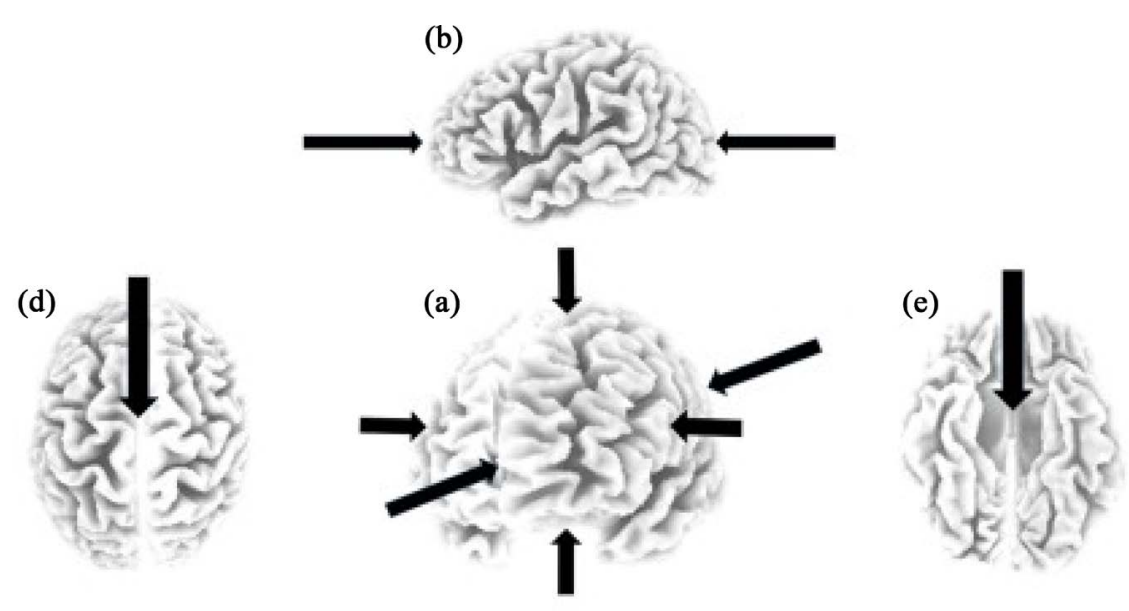

(c)

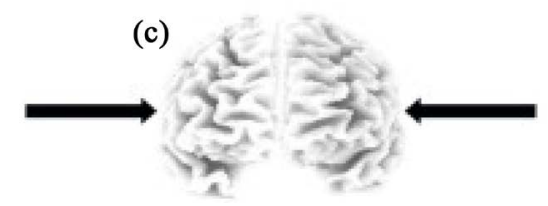

Figure 2. sLORETA template figures with arrows indicating the orientation of the PMT device during measurement procedure. All planes of space are represented (a) including rostral-caudal (b), left-right (c), as well as dorsal (d) and ventral (e) planes of space. 
Sagittal brain measurements of both left and right hemispheres were recorded over both the lateral and medial aspects (Figure 3) while having the photomultiplier unit suspended and within $9 \mathrm{~cm}$ between the aperture and the brain sample. The lateral aspect of both hemispheres was recorded by placing the sagittal brain on its medial aspect face down onto folded paper towel in the center of the box. The medial aspect was recorded by placing the sagittal section in the box with the lateral aspect face down. Baseline recordings were taken with the photomultiplier tube facing downward into the box, with no sagittal brain present.

Coronal sections were measured with the photomultiplier tube suspended above the tissue culture dish. Either the left or the right hemisphere was wrapped in a black nitrile glove (Figure 4). The photomultiplier unit was positioned over the exposed hemisphere when photon count recordings were ongoing. Baseline measures were taken with the EFA solution in the dish and without coronal brain samples present.

\subsection{Analyses}

All data were imported into IBM SPSS Statistics v20 and analyzed. Time series data were Z-scored and spectral analyzed (SPECTRA) and then averaged into the respective conditions. Because the sampling was 50 times per sec (25 ms increments) the Nyquist Limit for the spectral power densities (SPD) for the amplitude variations was $25 \mathrm{~Hz}$. All spectral analyzed data were then computed into binned intervals to analyze frequency differences employing mean comparisons and analysis-of-variance (ANOVA) measures. Averages (means) for photon counts per $25 \mathrm{~ms}$ from each recording were grouped into respective conditions in each experiment and analyzed through paired t-tests and ANOVA measures.

Because there was day-to-day variation in the photon counts for some regions of the brain but not for background levels or other brain regions we examined the potential contribution from geomagnetic fluctuations upon the variance of photon counts. The maximum global $\mathrm{K}$ indices obtained from the Boulder Station for the day were downloaded from the appropriate website. These $\mathrm{K}_{\mathrm{p}}$ values ranged primarily from 1 to 4 (there was one 8) during the 8 different days of photon measurement from the human brain samples. In previous experiments we [15] [16] had demonstrated quantitative equivalents between biophoton emissions from brain activity and very small and proximal shifts in the earth's magnetic field.

\section{Results}

\subsection{Whole-Brain}

A factor analysis with varimax rotation revealed 2 components, cumulatively explaining $77 \%$ of the variance.
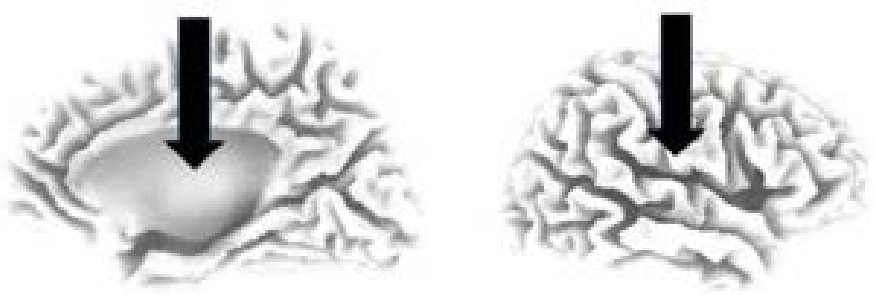

Figure 3. sLORETA 3D template representation of the medial (left) and lateral (right) aspects of a right sagittal section. Arrows indicate direction of the PMT during recordings.

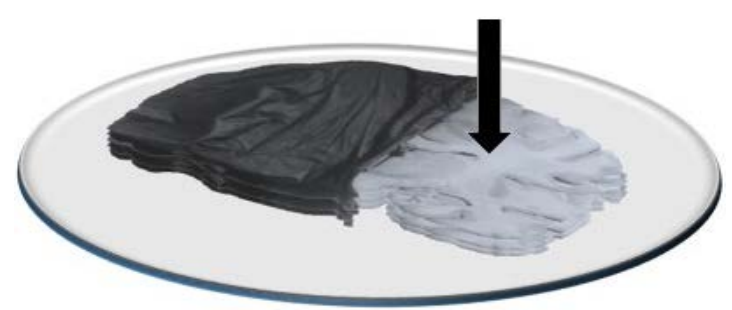

Figure 4. Coronal brain slices were fitted with a black nitrile glove, and placed within plastic tissue culture dishes filled with EFA solution. The right hemisphere is exposed. An arrow displays direction of the PMT during recording over the exposed hemisphere. 
The first factor, with factor loading scores greater than 0.5 , consisted of photon counts from caudal $(0.91)$, left $(0.98)$ right $(0.95)$, dorsal $(0.80)$, and ventral $(0.80)$ surfaces. The second factor consisted of background $(0.66)$ and rostral (0.76) photon counts. Paired t-tests revealed that within-brain significant differences could be identified when comparing left $(M=150.64, \mathrm{SEM}=5.40)$ and ventral $(\mathrm{M}=140.01, \mathrm{SEM}=4.55)$ photon counts, $\mathrm{t}(11)$ $=3.17, \mathrm{p}<0.01, \mathrm{r}^{2}=0.48$. Higher numbers of photon counts were recorded for the rostral $(\mathrm{M}=159.36, \mathrm{SEM}=$ 5.76) regions relative to ventral $(M=140.01, S E M=4.55)$ counts, $t(11)=2.86, p<0.05, r^{2}=0.43$. Rostral photon counts were also higher relative to dorsal $(\mathrm{M}=142.83$, $\mathrm{SEM}=4.86)$ photon counts, $\mathrm{t}(11)=2.58, \mathrm{p}<$ $0.05, r^{2}=0.38$. Figure 5 demonstrates these effects.

Over the range ( 0 to $25 \mathrm{~Hz}$ ) of the amplitude fluctuations the SPDs were not random. There was an obvious increase within a sustained band between 7 and $9 \mathrm{~Hz}$. Narrow-band factor analysis (varimax rotation) of photon count spectral power densities for the $7 \mathrm{~Hz}-9 \mathrm{~Hz}$ interval revealed four components that cumulatively accommodated $70 \%$ of the variance. The first component, explaining $20 \%$ of the variance, consisted of rostral $(0.71)$, caudal $(0.71)$, and left $(-0.54)$ photon counts. The second component, explaining $\sim 18 \%$ of the variance, consisted of baseline (0.79) and right hemisphere $(0.78)$ photon counts. This component displayed a conspicuous ( $z>4.5) 7.9 \mathrm{~Hz}-8 \mathrm{~Hz}$ peak (Figure 6). The third component, explaining 16\% of the variance, consisted of ven-

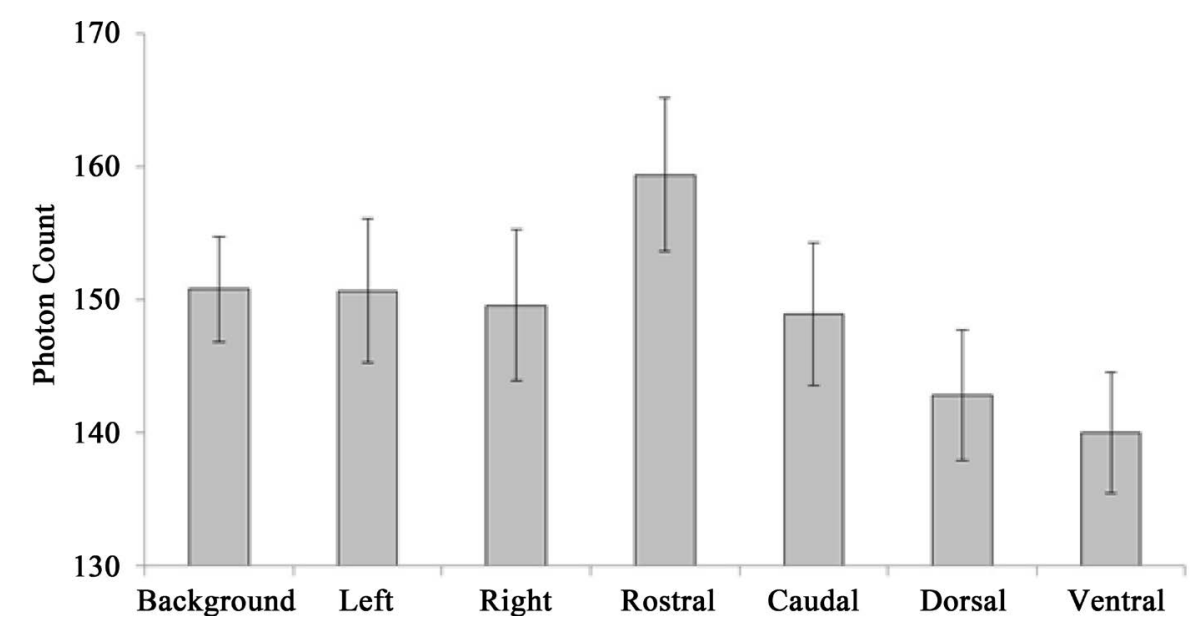

Figure 5. Photon counts obtained over various exposure aspects of whole human brain specimens including background values.

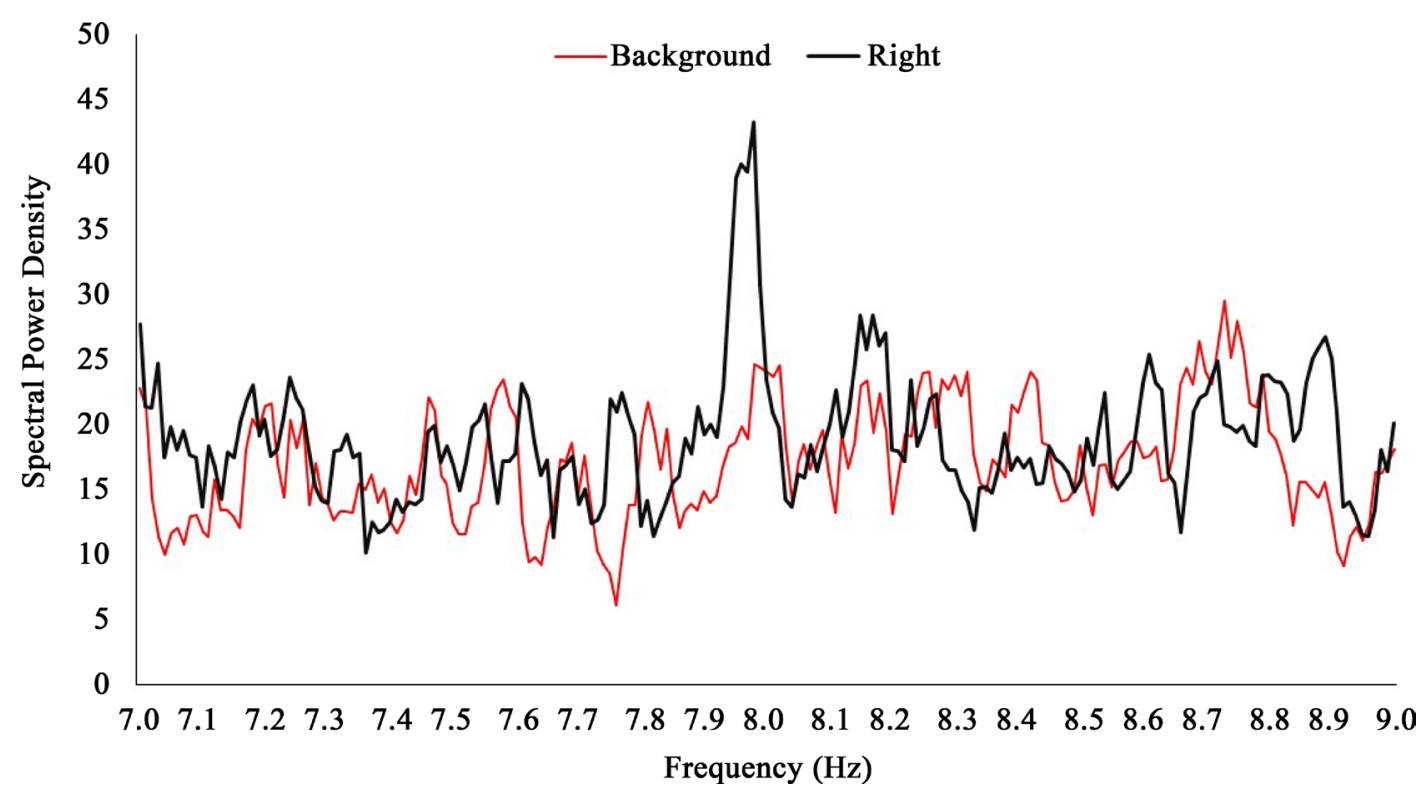

Figure 6. SPDs within the $7 \mathrm{~Hz}-9 \mathrm{~Hz}$ band associated with background photon counts and those obtained over the right hemisphere of whole human brain specimens. 
tral (0.87) and left (0.52) photon counts. The fourth and final component consisted of a single loading, dorsal $(0.91)$ photon counts, explained the final $\sim 16 \%$ of the variance.

\subsection{Light-Deprived Whole-Brain}

If the whole-brain specimens had been placed in a hyper-darkened chamber (that was also a Faraday cage and acoustic chamber) for a week there were conspicuously lower photon counts over the ventral aspect of the brain $(M=132.50, \mathrm{SEM}=4.82)$ that differed significantly from those obtained over the left hemisphere $(\mathrm{M}=149.76$, $\mathrm{SE}=5.62)$, right hemisphere $(\mathrm{M}=145.35, \mathrm{SEM}=4.42)$, rostrum $(\mathrm{M}=151.85, \mathrm{SEM}=7.32)$, caudal aspect $(\mathrm{M}$ $=144.13, \mathrm{SEM}=3.88)$, and dorsal aspect $(\mathrm{M}=149.00, \mathrm{SEM}=5.16)$. The effect sizes, the amount of variance accommodated, ranged between $51 \%$ and $68 \%$. Photon counts obtained over the rostral pole were higher relative to background values $(\mathrm{M}=139.66, \mathrm{SEM}=6.36), \mathrm{t}(8)=3.66, \mathrm{r}^{2}=0.63$. These results are presented in Figure 7 .

\subsection{Sagittal Brain}

Though no statistically significant differences in photon counts could be identified within hemispheres, there were clear and statistically significant between-hemisphere differences. They could be identified when medial and lateral surfaces were compared. Photon counts over the lateral surface of the sagittal sections were higher over the right hemisphere $(\mathrm{M}=136.65, \mathrm{SEM}=3.50)$ relative to the left $(\mathrm{M}=128.42, \mathrm{SEM}=1.58), \mathrm{t}(5)=2.91$, $\mathrm{p}<0.05, \mathrm{r}^{2}=0.63$. Photon counts over the medial surface of the sagittal sections were higher within the right hemisphere $(M=138.62, \mathrm{SEM}=4.24)$ relative to the left $(\mathrm{M}=127.10, \mathrm{SEM}=1.13), \mathrm{t}(5)=2.74, \mathrm{p}<0.05, \mathrm{r}^{2}=$ 0.60 . The lateral surface of the right hemisphere was associated with more photon counts relative to the medial surface of the left hemisphere, $\mathrm{t}(5)=3.52, \mathrm{p}<0.05, \mathrm{r}^{2}=0.71$. These results are presented in Figure 8 .

A plot of the right hemispheric SPDs between $7 \mathrm{~Hz}$ and $9 \mathrm{~Hz}$ revealed a number of peaks separated by increments of about $0.3-0.5 \mathrm{~Hz}$. One of these peaks centered around $8.1 \mathrm{~Hz}$ (Figure 9). The difference in power for the $8.1 \mathrm{~Hz}$ peak was equivalent to a z-score of 2.37 and was only exceeded by one peak centered around $8.8 \mathrm{~Hz}$. Its amplitude compared to all others was equivalent to a $z$-score of 3 . This pattern of significant increases in power within the $7 \mathrm{~Hz}$ to $9 \mathrm{~Hz}$ was not evident for left hemispheric SPDs within the same range (Figure 10). These spectral differences are noted despite clear overlap in mean photon counts as indicated in Figure 11.

In addition to simple hemispheric effects, a factor analysis employing varimax rotation involving SPDs computed within the $7 \mathrm{~Hz}-9 \mathrm{~Hz}$ range revealed (loading coefficients in parentheses) that left-medial $(0.71)$ and right-medial SPD (-0.72) loaded on the first of two components which cumulatively explained $61 \%$ of the variance. SPDs derived from photon counts obtained over the lateral surface of the left hemisphere loaded on the second factor (0.87) alone. SPDs derived from photon counts obtained over lateral surface of the right hemisphere did not load on either factor.

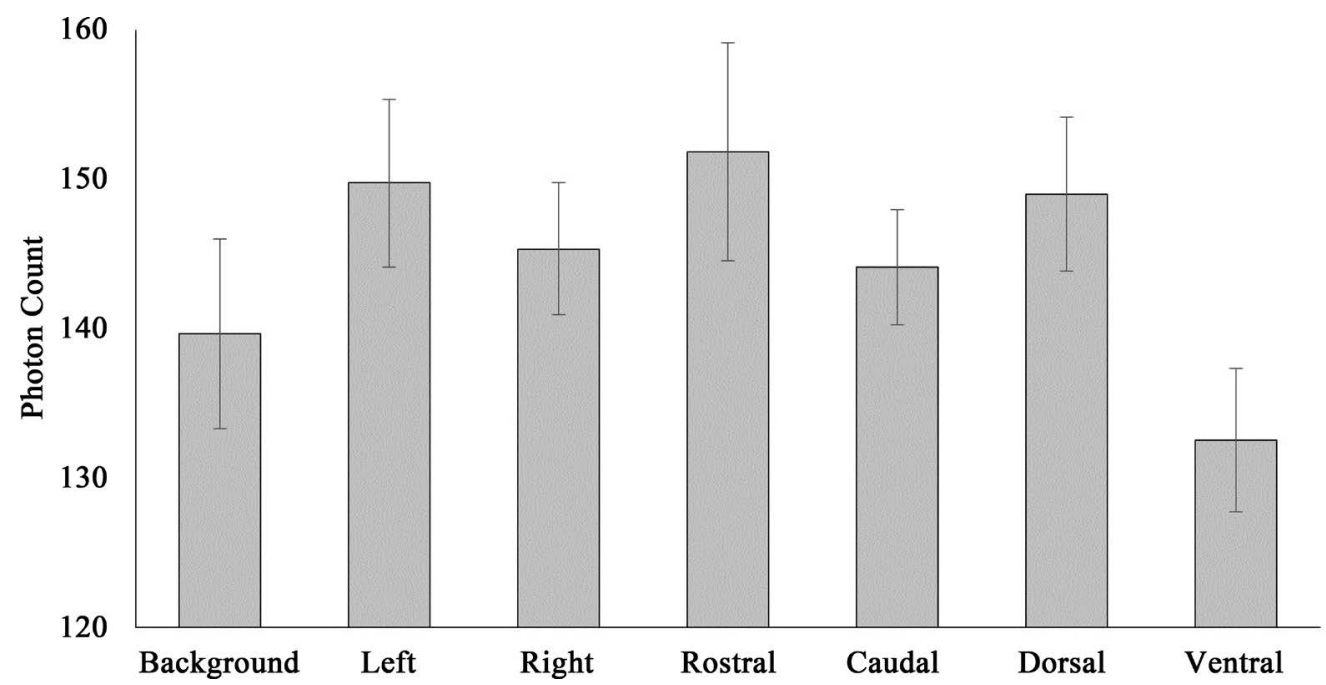

Figure 7. Photon counts obtained over various exposure aspects of light-deprived, whole human brain specimens including background values. 


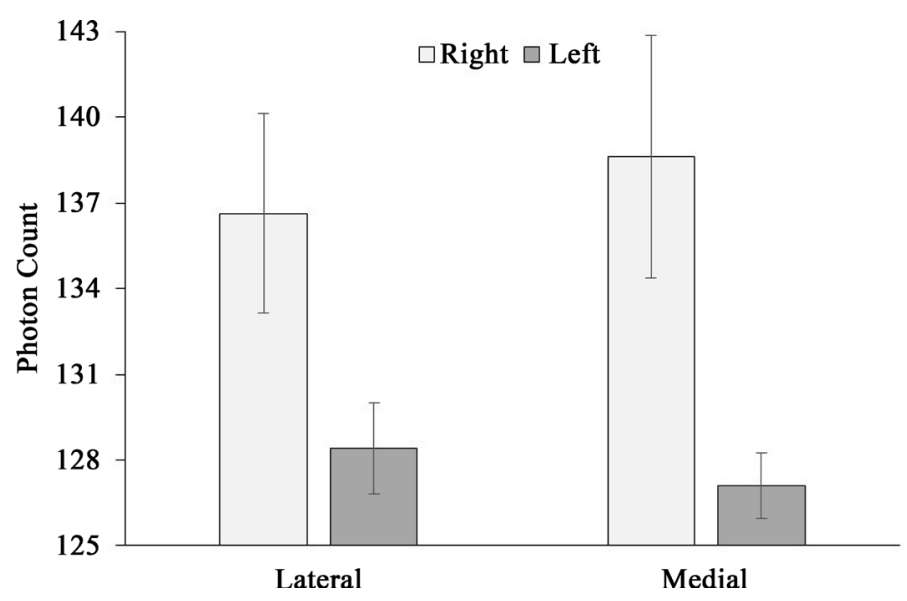

Figure 8. Photon counts as a function of medial-lateral surface aspect exposure obtained over right (light) and left (dark) hemispheres of sagittal sections.

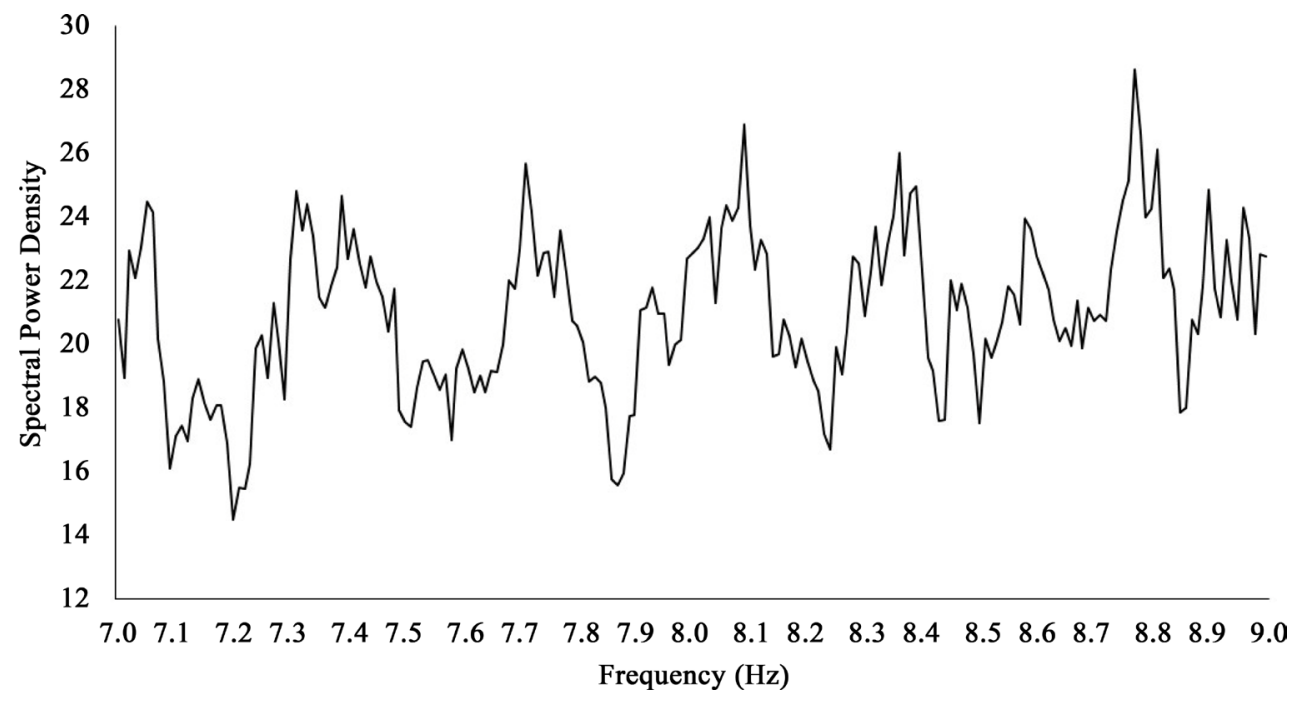

Figure 9. Average SPDs associated with photon counts over right hemispheric medial and lateral surfaces of sagittal sections within the $7 \mathrm{~Hz}-9 \mathrm{~Hz}$ band.

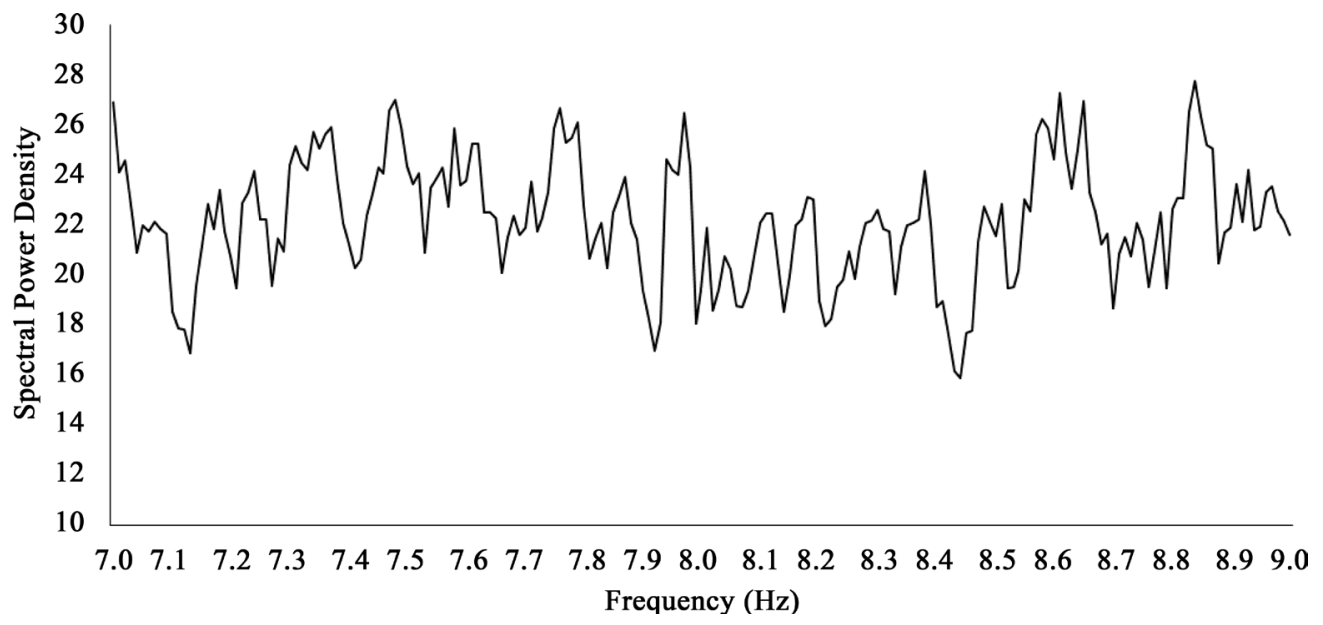

Figure 10. Average SPDs associated with photon counts over left hemispheric medial and lateral surfaces of sagittal sections within the $7 \mathrm{~Hz}-9 \mathrm{~Hz}$ band. 


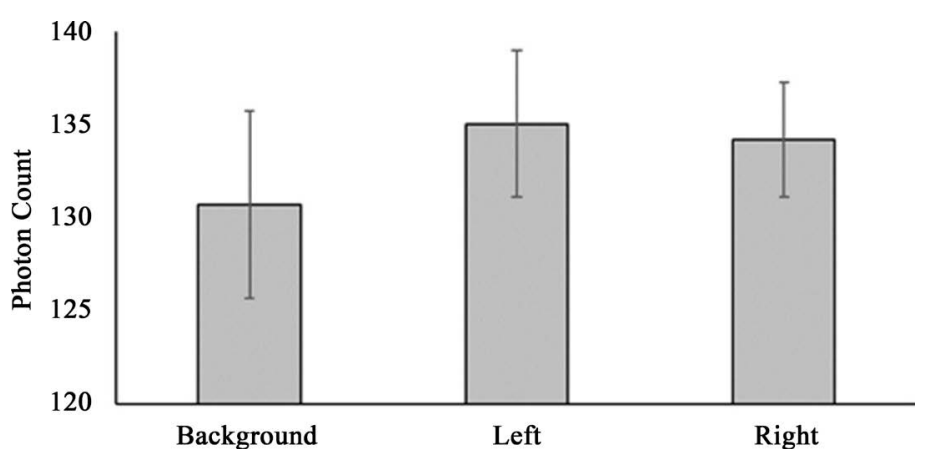

Figure 11. Mean photon counts obtained over left and right hemispheres of coronal human brain sections including background values. Means and standard errors are provided.

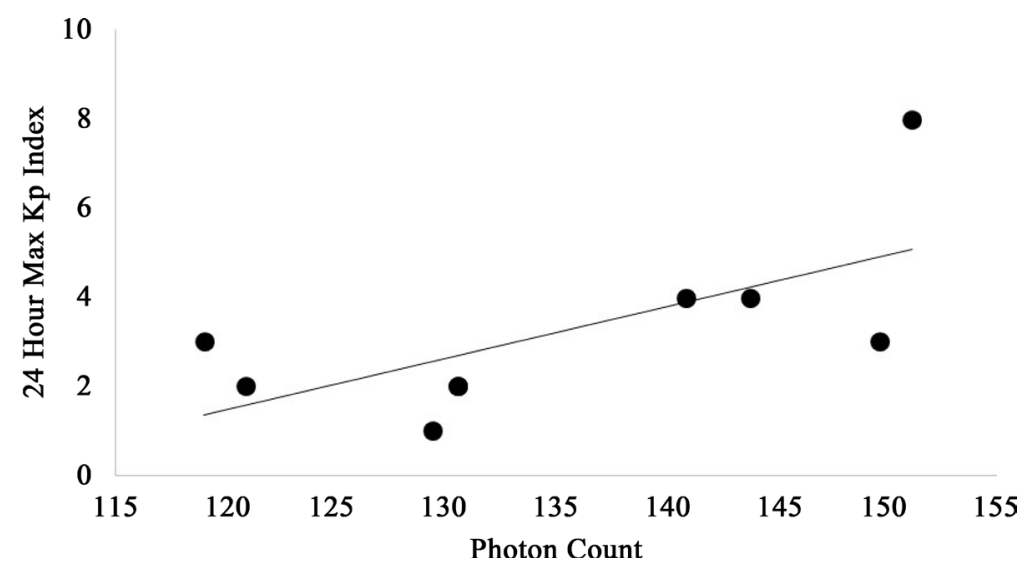

Figure 12. Significant positive correlation between 24 hour maximum Kp values and mean photon counts obtained over the left hemisphere of coronal sections of human brain.

However a strong correlation $($ rho $=0.88)$ between photon counts measured over left and right hemispheres was clearly evident. The magnitude of this correlation coefficient if it were a parametric value would suggest that $\sim 23 \%$ of the variance associated with photon emissions could not be explained by this powerful interhemispheric relationship and instead would be associated with some other source of variance.

The regression line in Figure 12 for the left hemispheric photon counts as a function of the maximum global $\mathrm{K}_{\mathrm{p}}$ index on the day of the photon measurements indicated a range between 120 and 150 counts per $20 \mathrm{~ms}$ and range of 1 to $4 \mathrm{~K}_{\mathrm{p}}$ units. According to Campbell's classic text [17], a $\mathrm{K}_{\mathrm{p}}$ of 1 is a variation (of the most disturbed component of the geomagnetic field) of about $4 \mathrm{nT}$ while a $\mathrm{K}_{\mathrm{p}}$ of 4 would be about $27 \mathrm{nT}$. Energy (E) within a volume immersed within a magnetic field can be calculated from:

$$
\mathrm{E}=\mathrm{B}^{2}(2 \mu)^{-1} m^{3}
$$

where B is the strength of the field; $\mu$ is magnetic permeability $\left(4 \pi \times 10^{-7} \mathrm{~N} \cdot \mathrm{A}^{-2}\right)$ and $\mathrm{m}^{3}$ is the volume of the sample. A difference of about $23 \mathrm{nT}$ within a volume of the coronal section of the left hemisphere $(50 \mathrm{cc}$ to 100 cc or $5 \times 10^{-5}$ to $10^{-4} \mathrm{~m}^{3}$ ) is $\sim 10^{-14}$ Joules.

On the other hand, the shift in photon counts $(150$ - 120) would be about 30 counts per $25 \mathrm{~ms}$ or 750 photons per s. Assuming a mid-value of $\sim 5 \times 10^{-19} \mathrm{~J}$ per photon the increase in photon energy output between the least and most geomagnetic activity and energy (excluding the outlier) within the volume of the sections would be 3.8 $\times 10^{-16} \mathrm{~J}$ per s per $\mathrm{cm}^{-2}$ (the width of the aperture). If this area were expanded over the volume of the section the total energy would be in the order of $10^{-14} \mathrm{~J}$. This would be within the same order of magnitude as the "intrinsic" energy within the brain section volume associated with the increased geomagnetic variations.

\section{Discussion}

The results of the measurements indicate that the human brain generates differential numbers of photons that 
may reveal some intrinsic structural capacity that remains after clinical death and following protracted fixation. Although the differences in photon counts measured from the rostral compared to the ventral regions of the whole brain were about 20 counts per $20 \mathrm{~ms}$ ( 1000 photons per s), the discrepancy may be significant. This is equivalent to about $5 \times 10^{-16} \mathrm{~J}$ per s $(\mathrm{W})$ and considering the aperture of the photomultiplier unit would be about $2 \times 10^{-12} \mathrm{~W} \cdot \mathrm{m}^{-2}$. This order of magnitude has been considered a persistent flux density that we have measured with both analogue and digital units. The value, which is about a factor of 10 above the background flux density of cosmic rays at ground level [14] and is meaningful, is indicated by its consistent inverse correlation with shifts in ambient geomagnetic fields in the order of $1 \mathrm{nT}$ [15] [16].

If the emissions were "random", one would not expect the two distinct factor loadings for the amplitude fluctuations of the spectral power densities. They would have been less influenced by the nuances of net photon numbers. One involved shared profiles of photon power densities over the rostral, caudal, and left regions of the whole brain. The other involved the shared variance between the power densities over the right hemisphere and the baseline (no tissue present) conditions. This discrepancy is important for two reasons. First it suggests that there is a structural difference that is still present within the two human cerebral hemispheres many years after clinical death within appropriately fixed tissue. The greater number of photons emitted from the right hemisphere compared to the left was evident for the coronal and sagittal sections. The right hemisphere emitted about 500 "spontaneous" photons per s more than the left hemisphere.

Second, the conspicuous peak between 7.9 and $8 \mathrm{~Hz}$ for that power density is remarkably similar to both the peak range of power within the living quantitative electroencephalographic profile of older people (which was the likely source of these brains) as well as the fundamental frequency of the Schumann Resonance that is generated between the earth's surface and the ionosphere [18]. We measure the Schumann Resonances daily. We have suggested there is an intrinsic organization within the right hemisphere that facilitates intercalation between this region of the living human brain and the Schumann power spectra. Recently Saroka et al. [19] and Persinger and Saroka [20] reported quantitative evidence for this relationship as well as the theoretical bases for the concordance in large populations.

There was quantitative evidence that the whole brain deprived of exposure to ambient light $\left(\sim 10^{2}\right.$ lux $)$ in the standard laboratory setting for about $12-18 \mathrm{hrs}$ per day (before the lights are extinguished for the night) emitted about 5 to 8 more photons per $20 \mathrm{~ms}$ than the same whole brains that were maintained in the hyper-dark environment for a week. Although potentially spurious, we suggest that the energy associated with this small numbers of photons is equivalent to a wavelength that overlaps with the Bohr orbit within a temporal frame ( $\sim 20$ to $25 \mathrm{~ms}$ ) coupled with the recurrent rostral-to-caudal transcerebral cortical fields associated with consciousness [21] is relevant. This effect was repeated several times. Although the differential values for photon counts from the different planes of the whole brains remained relatively consistent the absolute numbers of photons were less in the brains that had been deprived of ambient light. The diminishment of photons when the brains had been exposed for a week in the hyper-dark settings approached the likely background level associated with cosmic ray flux density at sea level.

From another perspective, the measurements suggest that fixed brain tissue in the laboratory exposed to ordinary lighting has the capacity to "store" photons in some manner, perhaps analogous to Popp's virtual concepts [1], that is then re-emitted within the hyperdark setting. Karbowski et al. [22] have shown that living melanoma cells in culture display this capacity. They found that when pulsed $470 \mathrm{~nm}$ (LED) light was coupled to a specific weak magnetic field (designed from geomagnetic sudden impulses) the cells maintained the photon energy and re-emitted it during the subsequent 30 to $60 \mathrm{~min}$ after the cells had been removed from the fields and the light but maintained in the dark. The total energy associated with the delayed photon emissions was directly related to the energy available within the cellular volume from the intensities of the applied magnetic fields. One experiment that might demonstrate an equivalent effect within fixed, dead human brain tissue would involve exposure to appropriately patterned weak magnetic fields that simulate natural geomagnetic activity with concomitant same-patterned pulses of specific LED (light emitting diodes) photons. The re-emission of these wavelengths in a manner similar to the Karbowski et al. [22] experiments would support the capacity for the fixed human brain to retain potentially photonic information.

A surprising result in the present study was the statistically significant correlation between the numbers of photon emissions from the left hemispheres only for the various sessions and the indices of global geomagnetic activity. Over the median range of approximately $27 \mathrm{nT}$ there was an increase of about 30 counts per $20 \mathrm{~ms}$. Calculations indicated that the photonic energy released was in the same order of magnitude as the energy that 
would have occurred within the volume of the brain from the increased geomagnetic variation. This correlation only occurred from the photons that were measured over the left hemisphere and not the right hemisphere. The application of either weak, physiologically patterned extremely low frequency magnetic fields [23] or 0.1 to 1 GHz microwave sources [24] over the left hemisphere but not the right hemisphere has been shown experimentally to modify the power within the alpha range of electroencephalographic activity in human beings. The power densities in the latter experiments were equivalent to $\sim 10^{-11} \mathrm{~W} \cdot \mathrm{m}^{-2}$. The normal discrepancy between hemispheres in living brains reduces the probability the effects we observed in the present experiments were adventitious and suggests that intrinsic differences in cerebral structure, still present after years of fixation, might respond differently to environmental energy sources. Of the approximately 176 sulci and gyri that comprise the left and right hemispheres of the human brain only a few, such as the central sulcus, Sylvian fissure on the lateral side, the calcarine sulcus and the cingulate sulcus are similar in both hemispheres [25].

There are significant implications for the interface between medicine and neuroscience. For example we have found that although microtubule preparations exposed to weak, physiologically patterned magnetic fields may exhibit increased photon emissions the more critical feature was the shifts in spectral power patterns of photon flux densities. These patterns represented information being transferred between microtubules. Trushin [11] has suggested photons may be a fundamental means by which bacteria, one of the most persistent and universal life forms, "communicate". Recently we found that cultures of malignant cells from various types of organs exhibited unique photon spectral density profiles that could differentiate their origins and could be employed to completely discriminate non-malignant and malignant populations. Bokkon [26] had postulated that sequences of visual imagery within the human cerebrum were photon fields rather than phenomenological correlates of action potentials. He and his colleagues [9] [27] calculated there were more photons within neurons and that those that were emitted may contain representative information. Experiments by Dotta et al. [7] showed that instructing subjects sitting in hyperdarkness to imagine white light was associated with increased photon emissions from their right (but not their left) hemispheres. These specific increases did not occur when the same subjects engaged in banal ideation. The potential for discerning what type of information can be extracted from fixed cerebrums must still be examined empirically.

\section{References}

[1] Popp, F.-A. (1979) Photon Storage in Biological Systems. In: Popp, F.-A., Becker, G., Koenig, H.L. and Peschka, W., Eds., Electromagnetic Bio-Information, Urban \& Schwarzenberg, Munich, 123-148.

[2] Popp, F.-A. (1988) Biophoton Emission. Experientia, 44, 543-544. http://dx.doi.org/10.1007/BF01953300

[3] Popp, F.-A., Li, K.H., Mei, W.P., Galle, M. and Neurohr, R. (1988) Physical Aspects of Biophotons. Experientia, 44, 576-585. http://dx.doi.org/10.1007/BF01953305

[4] Tilbury, R.N. and Quickenden, T.I. (1988) Spectral and Time Dependence Studies of the Ultra-Weak Bioluminescence Emitted by the Bacterium Escherichia coli. Photochemistry and Photobiology, 47, 145-150. http://dx.doi.org/10.1111/j.1751-1097.1988.tb02704.x

[5] Dotta, B.T., Buckner, C.A., Cameron, D., Lafrenie, R.F. and Persinger, M.A. (2011) Biophoton Emissions from Cell Cultures: Biochemical Evidence for the Plasma Membrane as the Primary Source. General Physiology and. Biophysics, 30, 301-309.

[6] Kobayashi, M., Takeda, M., Sato, T., Yamakazi, Y., Kaneko, K., Ito, K.-I., Kato, H. and Inaba, H. (1999) In Vivo Imaging of Spontaneous Ultra-Weak Photon Emission from a Rat's Brain in Vivo. Journal of Neuroscience Methods, 34, 103-113.

[7] Dotta, B.T., Saroka, K.S. and Persinger, M.A. (2012) Increased Photon Emissions from the Head While Imagining Light in the Dark Is Correlated with Changes in Electroencephalographic Power: Support for Bokkon's Biophoton Hypothesis. Neuroscience Letters, 513, 151-154. http://dx.doi.org/10.1016/j.neulet.2012.02.021

[8] Van Wijk, R., Van Wijk, E.P.A. and Bajpai, R.P. (2006) Photocount Distribution of Photons Emitted from Three Sites of the Human Body. Journal of Photochemistry and Photobiology B: Biology, 84, 46-45. http://dx.doi.org/10.1016/j.jphotobiol.2006.01.010

[9] Bókkon, I., Salari, V., Tuszynski, J.A. and Antal, I. (2010) Estimation of the Number of Biophotons Involved in the Visual perception of a Single-Object Image: Biophoton Intensity Can Be Considerably Higher inside Cells than outside. Journal of Photochemistry and Photobiology B: Biology, 100, 160-166. http://dx.doi.org/10.1016/j.jphotobiol.2010.06.001

[10] Fels, D. (2009) Cellular Communication through Light. PLos ONE, 4, e5086. 
http://dx.doi.org/10.1371/journal.pone.0005086

[11] Trushin, M.V. (2004) Light-Mediated Conversation among Microorganisms. Microbiological Research, 159, 1-10. http://dx.doi.org/10.1016/j.micres.2003.11.001

[12] Persinger, M.A. (2012) Annual Variation of Local Photon Emissions' Spectral Power within the mHz Range Overlaps with Seismic-Atmospheric Acoustic Oscillations. International Journal of Geosciences, 3, 192-194. http://dx.doi.org/10.4236/ijg.2012.31021

[13] Persinger, M.A., Lafreniere, G.F. and Dotta, B.T. (2012) Marked Increases in Background Photon Emissions in Sudbury Ontario more than One Week before the Magnitude $>8.0$ Earthquakes in Japan and Chile. International Journal of Geosciences, 3, 627-629. http://dx.doi.org/10.4236/ijg.2012.33062

[14] Konig, H.L., Kreuger, A.P., Lonag, A.P. and Sonning, W. (1981) Biologic Effects of Environmental Magnetism. Springer-Verlag, New York. http://dx.doi.org/10.1007/978-1-4612-5859-9

[15] Hunter, M.D., Mulligan, B.P., Dotta, B.T., Saroka, K.S., Lavallee, C.F., Koren, S.A. and Persinger, M.A. (2010) Cerebral Dynamics and Discrete Energy Changes in the Personal Physical Environment during Intuitive-Like States and Perceptions. Journal of Consciousness Exploration \& Research, 1, 1179-1197.

[16] Persinger, M.A., Dotta, B.T., Karbowksi, L.M. and Murugan, N.J. (2015) Inverse Relationship between Photon Flux Densities and NanoTesla Magnetic Fields over Cell Aggregates: Quantitative Evidence for Energetic Conservation. FEBS Open Bio, 5, 413-418. http://dx.doi.org/10.1016/j.fob.2015.04.015

[17] Campbell, W.H. (1997) Introduction to Geomagnetic Fields. Cambridge University Press, Cambridge.

[18] Nickolaenko, A. and Hayakawa, M. (2014) Schumann Resonance for Tyros. Springer, Tokyo. http://dx.doi.org/10.1007/978-4-431-54358-9

[19] Saroka, K.S., Vares, D.A.E. and Persinger, M.A. (2016) Similar Spectral Power Densities within the Schumann Resonance and a Large Population of Quantitative Electroencephalographic Profiles: Supportive Evidence for Koenig and Pobachenko. PLOS ONE, 11, e0146595. http://dx.doi.org/10.1371/journal.pone.0146595

[20] Persinger, M.A. and Saroka, K.S. (2015) Human Quantitative Electroencephalographic and Schumann Resonance Exhibit Real-Time Coherence of Spectral Power Densities: Implications for Interactive Information Processing. Journal of Signal and Information Processing, 6, 153-164. http://dx.doi.org/10.4236/jsip.2015.62015

[21] Llinas, R.R. and Ribardy, U. (1993) Coherent 40-Hz Oscillations Characterizes Dream State in Humans. Proceedings of the National Academy of Sciences of the United States of America, 90, 2078-2081. http://dx.doi.org/10.1073/pnas.90.5.2078

[22] Karbowski, L.M., Murugan, N.J. and Persinger, M.A. (2016) Experimental Evidence that Specific Photon Energies Are "Stored" in Malignant Cells for an Hour: The Synergism of Weak Magnetic Field-LED Wavelength Pulses. Biology and Medicine, 8, BM-162-16.

[23] Persinger, M.A. (1999) Increased Emergence of Alpha Activity over the Left but Not the Right Temporal Lobe within a Dark Acoustic Chamber: Differential Response of the Left but Not the Right Hemisphere to Transcerebral Magnetic Fields. International Journal of Psychophysiology, 34, 163-169. http://dx.doi.org/10.1016/S0167-8760(99)00069-0

[24] Stocklin, P.L. and Stocklin, B.F. (1981) Low Power Microwave Effects on the Human Electroencephalogram: Supporting Results of Bise. Physiology, Chemistry and Physics, 13, 175-177.

[25] Van Essen, D.C. and Drury, H.A. (1997) Structural and Functional Analyses of Human Cerebral Cortex Using a Surface-Based Atlas. The Journal of Neuroscience, 17, 7079-7102.

[26] Bokkon, I. (2005) Dreams and Neuroholography: An Interdisciplinary Interpretation of Development of the Homeotherm State in Evolution. Sleep and Hypnosis, 7, 61-76.

[27] Bokkon, I., Dai, J. and Antal, I. (2010) Picture Representation during REM Dreams: A Redox Hypothesis. Biosystems, 100, 79-86. http://dx.doi.org/10.1016/j.biosystems.2010.01.005 